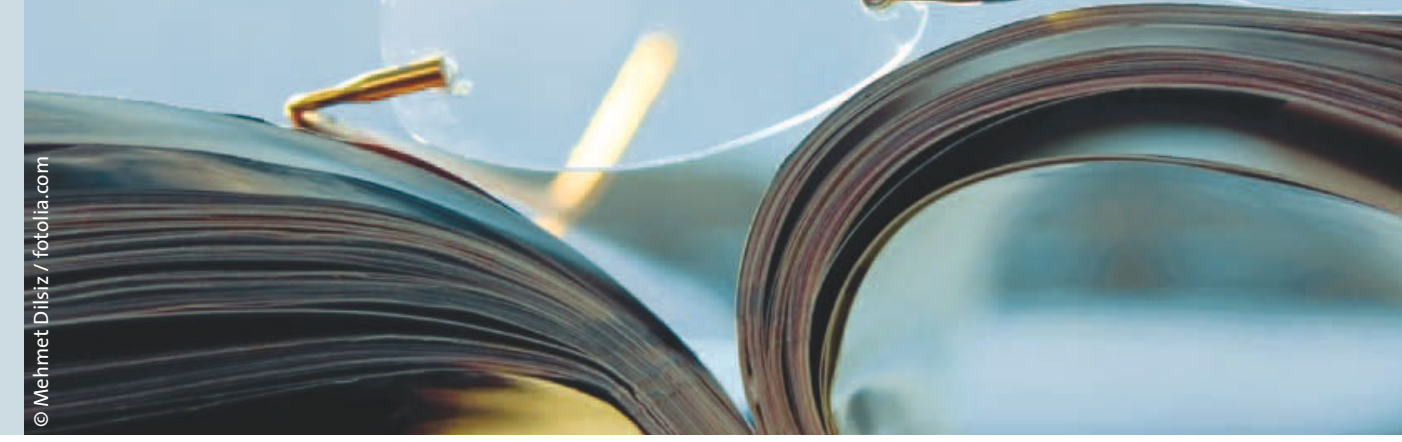

\title{
Botulinumtoxin A bei überaktiver Blase: Dosis entscheidend
}

\begin{abstract}
Botulinumtoxin A hat schon vor Längerem seine Wirksamkeit bei der Behandlung der idiopathisch überaktiven Blase und Dranginkontinenz bewiesen. Doch welche Dosis des Neurotoxins muss in den M. detrusor vesicae injiziert werden, damit die Behandlung wirksam und gleichzeitig sicher ist?
\end{abstract}

P atienten mit überaktiver Blase stehen vor mehreren Therapieoptionen. Eine sind oral einzunehmende Anticholinergika, die zwar die Symptomatik mildern, aber auch Nebenwirkungen mit sich bringen können. Seit 2000 erstmals über Botulinumtoxin A als vielversprechendes Therapeutikum bei neurogener Detrusorüberaktivität berichtet wurde, hat diese Substanz in verschiedenen Studien ihre Wirksamkeit unter Beweis gestellt, auch bei idiopathisch überaktiver Blase und/oder Detrusorüberaktivität mit Harninkontinenz.

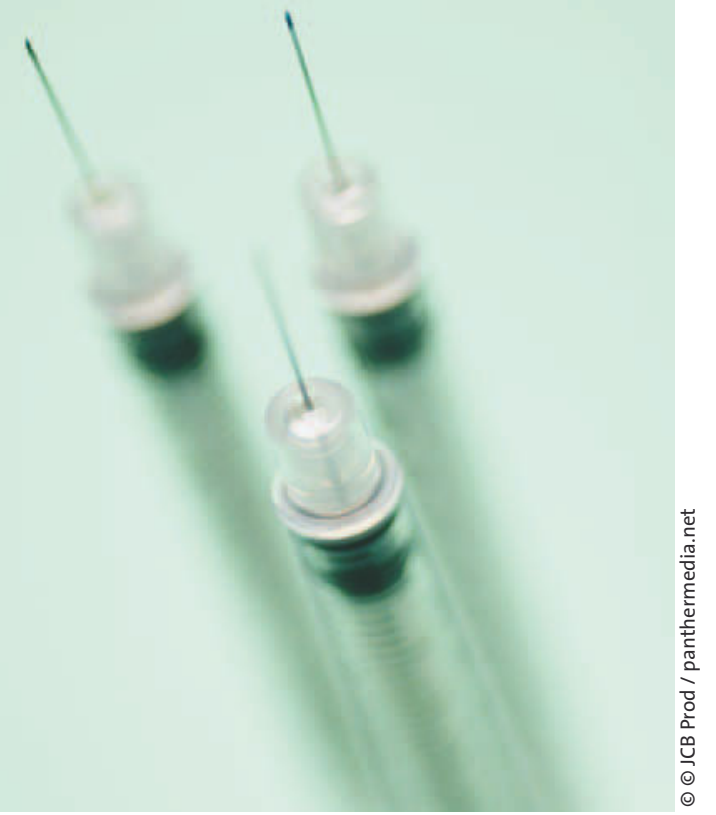

Welche Botulinumtoxindosis sollte bei Dranginkontinenz injiziert werden?
US-amerikanische Forscher testeten nun, welche Dosierung des Neurotoxins eine gute Wirkung und gleichzeitig größtmögliche Sicherheit garantiert. In die randomisierte doppelblinde multizentrische Studie wurden 272 Patienten mit idiopathisch überaktiver Blase und Harninkontinenz einbezogen. Alle Probanden litten unter acht oder mehr Dranginkontinenz-Episoden pro Woche und mussten achtmal oder öfter pro Tag Wasser lassen. Die Autoren injizierten den Teilnehmern entweder Placebo oder Onabotulinumtoxin A in einer Dosierung von 50, 100, 150, 200 oder $300 \mathrm{U}$ in den M. detrusor vesicae. Das Followup erfolgte nach sieben Tagen und in den Wochen 2, 6, 12, 18, 24, 30 und 36. Als primäres Wirksamkeitskriterium wurde bei jeder Nachuntersuchung die Zahl der Dranginkontinenz-Episoden pro Woche im Vergleich zu Studienbeginn erhoben. Als sekundäre Wirkkriterien hat man die Miktionshäufigkeit pro Woche, Harndrang und Nykturie sowie das Restharnvolumen erhoben. Als primären Endpunkt definierte man die Woche 12.

Eine dauerhafte Wirkung wurde bei allen Patienten erzielt, die mit $100 \mathrm{U}$ oder mehr Botulinumtoxin A behandelt wurden, bezogen auf alle primären und sekundären Wirksamkeitskriterien. In allen Verumgruppen verringerte sich die Zahl der Dranginkontinenz-Episoden bereits in Woche 2. Dabei schnitten während der gesamten Nachuntersuchungsperiode die Verumgruppen deutlich besser ab als die Placebogruppe. An Woche 12 betrug die durchschnittliche Differenz im Vergleich vor Therapie -17,4 in der Placebogruppe sowie -20,7, -18,4, $-23,0,-19,6$ in den Therapiegruppen mit $50,100,150,200$ und 300 U Botulinumtoxin A. Die Analyse der DosisWirkungs-Kurve ergab, dass sich ab einer Dosis von $150 \mathrm{U}$ die Wirksamkeit nicht mehr steigern lässt. Diese Ergebnisse spiegelten sich auch in den Lebensqualität-Parametern wider.

Die Parameter Restharnvolumen und Notwendigkeit zur zeitweisen Katheterisierung veränderten sich dosisabhängig. Nebenwirkungen wie Infektionen des Harntrakts und Harnverhalt waren unter Botulinumtoxin A im Vergleich zu Placebo deutlich häufiger zu beobachten.

Fazit: Onabotulinumtoxin A in einer Dosierung von $100 \mathrm{U}$ oder höher ist bei der Therapie der idiopathisch überaktiven Blase und Harndranginkontinenz dauerhaft wirksam. Dabei ist die 100 U-Dosis zu präferieren, denn bei dieser Dosierung fällt das Nutzen-Risiko-Verhältnis am günstigsten aus. Bei Patienten, die bereits mit einem oralen Anticholinergikum vorbehandelt sind, sehen die Autoren in der Botulinumtoxininjektion eine geeignete Alternative zu anderen minimal-invasiven Therapiemaßnahmen wie etwa der Neuromodulation und auch zur chirurgischen Harnblasenaugmentation.

$\mathbf{d k}$

Dmochowski R et al. Efficacy and Safety of OnabotulinumtoxinA for Idiopathic Overactive Bladder: A Double-Blind, Placebo Controlled, Randomized, Dose Ranging Trial. J Urology 2010, 184: 2416-22 